\title{
Impact of Safe Water and Improved Sanitation on Incidence/Prevalence of Typhoid Fever Globally
}

\author{
Article by Otojareri Kohwo Anthony \\ NHIS Unit, Federal Medical Centre, Bida, Niger State, Nigeria \\ Email: otojareri.kohwo@yahoo.com
}

\begin{abstract}
Typhoid fever is a systemic infection caused by the bacterium salmonella typhi. It occurs worldwide and more in developing countries. This study is a review of existing journals on typhoid fever is aimed to determine whether safe water usage or consumption and improved sanitation had an impact on the incidence/prevalence of typhoid fever globally.

A literature search is carried out reviewing current and existing journals on typhoid fever.

From the view, it was noted in most of the articles by various authors that safe water usage and improved sanitation impacted on incidence and prevalence of typhoid fever.

It is however recommended that with implementation of various public health strategies on safe water, improved sanitation and personal hygiene there will be a reduction in the incidence of typhoid fever in our environment.

Vacuration program on Typhoid should be stepped to further curb the high mortality rate from this water borne disease.
\end{abstract}

Keywords: Typhoid fever, safe water, unproved sanitation.

\section{Introduction}

Typhoid fever is a systemic infection caused by the bacterium salmonella enteric serotype Typhi (S. Typhi) John and Gumpet, 2010). It occurs worldwide and more in developing countries. It is a major cause of enteric disease in children in developing countries (Kariuki, 2008). It causes mortality in young adults in their productive life making it a disease with great socio economic impact. (Siddiqui, 1991).

The annual estimate incidence in Africa is about 400,000 cases, giving about 50 cases per 100,000 persons per year. The disease occurs commonly amongst school age children. Siddiqui, 1991 in a study on epidemiology patterns and control strategy, noted that there are 33 million case and 500,000 deaths from typhoid fever, annually occurring globally.

In the developing world, a worldwide incidence is $365 / 100,00(0.5 \%)$ whilst in countries as Japan and USA with a good reporting system, the annual incidence is $0.24-3.7 / 100,00$ population (Eddman and Levine, 1986). Typhoid fever occurs amongst the low and middle income class countries. The transmission of Typhoid fever is by ingestion of food or water contaminated with faces from carriers or expedients or infected persons.

The diagnosed of the diseases lies on laboratory confirmation. Bone mamor culture remains the goal standard diagnostic test. Though blood culture is a less sensitive method than bone manoro culture, blood culture is practically the first choice for patient diagnose. Serologic methods for diagnose still suffered from substantial limitation of both sensitivity and specificity (Muite and Crump, 2010).

Several researchers have carried out studies on the incidence/prevalence of typhoid fever with emphases on how to curb its increase with the employment of strategies as provision of safe water, improving sanitation, improving personal hygiene and vaccination etc. The object of the study is to carry out a literature search on the interest, to reviewing as many articles as possible to see if the impact of improving access, to portable water, improving sanitation with proper sewage treatment could help reduce the incidence of this disease.

Brieman et al., 2013, carried out a comparative study to assess the incidence of typhoid fever in an urban informal settlement and a rural area Kenya, found, a higher incidence in the 
urban area in which led to looking into the problems of overcrowding mineral access to safe water and poor hygiene practices. The results of study showed a very high incidence amongst urban children less than 10years old. These rates are comparable to studies carried out in Asia. Outcome of the study further stressed on use of vaccination coverage problem as a strategy to reduce incidence to typhoid fever.

A review of the burden of typhoid fever in Bangladesh by Mesbah Uddin Ahmed, noted that Typhoid fever is highly endemic in South East Asian countries with about 21.7 million new cases emerging each year. There are 216,000 annual death recorded with about 358 to 1100 per 100,000 annual attack rate. (Bullettin WHO, 2008). Also inferred from this study are risk factors associated with Typhoid fever in rural areas and they are poor sanitation, overcrowding, lack of safe water supply. These have help to design ways of prevention of typhoid fever, such as improvement in public Health Education, early detection and treatment of disease, hand washing, avoid drinking untreated water or eating of raw fruits and vegetable as well as administration of vaccine to high risk group.

Also as earlier noted from 1991 study on epidemiology patterns and control strategy in Typhoid fever, Siddiqui 1991 showed how the Typhoid can be controlled by provision of safe clear water, effective sewage disposal and prompt diagnosis and treatment of patients as well as asymptomatic carriers. In most endured region for typhoid fever, the poor segment of the population usually remains in areas underserved in terms of adequate water and sewage system. When these measures for disease control are enacted it will help to reduce the incidence of typhoid fever.

(Hatta et al., 2009) on risk factor for clinical typhoid fever in villages in rural south Sulaweisi, Indonesia, the author carried out a survey among all household head in five neighbouring villages in rural south - Sulawesi in Indonesia. He went to know the prevalence of typhoid fever and risk factors as demographic and behavioural for having typhoid. About 134 cases of typhoid fever were recorded of which $90 \%$ was diagnosed based on Clinical findings.

India pendent risk factors identified were consumption of uncooked vegetables, consumption of water with poor quality, use of water contaminated with conliform bacteria; not washing hands before eating and not using soap for washing hand. The results of this study again showed that absence of clean drinking water adequate sanitation education which focused on simple measures as hand washing and soap before eating or cooking before consumption may contribute to prevention of typhoid fever in rural areas. The building and use of pot toilet and construction of a water supply system should be prioritized to improve the health of the population.

On prevention of typhoid fever, an article posited on Wikipedia encyclopedia reviewed that sanitation and hygiene are important measures that can be taken to prevent typhoid. Typhoid can spread on environment where human faces or urine comes in contact with food or drinking water, and so careful food preparation and washing of hands are important in prevention of typhoid fever. In a similar article published in Microbewiks, on typhoid in China, vaccines were recommended for travelling outside China to US, Canada, Northern Europe, Austrians and New Zealand during epidemic outbreaks. As immunization is not always completely effective, travelers at risk should drink only boiled or bottled water and eat well- cooked food. Adequate water treatment, waste disposal and protection of food from contamination are important public health measurers. Carriers of typhoid according to the article must not be allowed to work as food handlers.

\section{Methodology}

A literature search outline was carried out reviewing articles that discussed typhoid fever and the impact of water treatment and improved sanitation of prevalence of typhoid. Most of the articles cited were critiqued after review and various findings gotten were discussed. 


\section{Findings and discussion}

Several years after the United Nation Millennium Declaration in its Eight Millennium Development Goal which stated and stressed the global importance of safe water, improved sanitation and hygiene for development, poverty reduction and health, the incidence of typhoid fever is still on the high side.

The literature noted in a study by Mesbah Ahmed that the incidence of Typhoid fever in Endemic South East Asian countries quoted about 21.7 million new cases occurring each year, with about $90 \%$ of this figure occurring in South East Asia, 216,000 deaths are recorded annually with about 358 to 11000 per 100,000 annual attack rate (WHO Bulletin, 2008).

The 1991 study on epidemiologic patterns and control strategies showed a similar high incidence figure. It estimated that 33 million cases and 500,000 deaths occurred annually throughout the developing world. Worldwide incidence was put at 365 per 100,000 and 540 per 100,000 in the developing world. This showed there is a higher incidence of Typhoid fever in the developing world as well as high death rate.

A similar high incidence/prevalence figure was mentioned in an Article posted on Wikipedia on Typhoid fever, showed that in 2010, 190,000 deaths were recorded which was high than the figure 137,000 deaths in 1990 from Typhoid fever.

Most of the studies/literatures reviewed in this literature search noted that poor sanitation, hygiene and absence of clean water contributed to increase in the disease. Overcrowding, eating of raw fruits and vegetable that is contaminated were also implicated as risk factors that causes increase in incidence of Typhoid fever.

Sameen Siddiqui (1991) in his study which supported the above mentioned risk factors noted that in most endemic region for Typhoid fever, the poorer segments of the population usually live in areas underserved in terms of adequate water and improved sewage systems. This study is similar to an article on health impacted caused by poor water and sanitation. The study commented that poor people in most developing countries have a great burden of diseases due to inadequate water supply, sanitation and hygiene.

Prevention of Typhoid fever can be well achieved if most of the risk factors are reversed or well handled. Most of the literature reviewed in this project noted that public health strategies are improvement in public health education, provision of portable water to most communities, stepping up improvement in sanitation, improved personal hygiene, early detection and treatment of cases as well as vaccination coverage in high risk area will have a great impact on reducing the incidence of prevalence/incidence of Typhoid fever.

\section{Conclusion}

With implementation of public health strategies on safe water, improved sanitation and personal hygiene, there will be a reduction in the incidence of Typhoid fever in our environment. Government in various countries should be on top gear with the implementation of Millennium Development Goal Eight in order to reduce the high mortality rate from Typhoid fever.

Vaccination programs on Typhoid should also be stepped up to further curb the high mortality rate from this water borne disease.

\section{References}

[1.] Condran G.A, Crimmins - Gardner E. (1978) Public Health measures and mortality in US Cities in the late nineteenth century. Human Ecology 6: 27- 54.

[2.] Crump J.A, Luby S.P et al (2004). The global burden of typhoid fever. Bull WHO82:346 - 353.

[3.] Cutter D, Milter G. (2005). The Role of Public Health improvement in health advances: the twentieth century United State Demographics 42: 1 - 22.

[4.] Edelman, R and Lerine, M.M (1986) Summary of an International Workshop on typhoid fever. Rev infect Dis. 8:329.

[5.] Esrey S.A, Feachem R.G, et al (1985). Intervention for the control of diarrheal diseases among young children; improving water supplies and excreta disposal facilities. Bull WHO 63, 757 - 772. 
South American Journal of Public Health

Volume 4, Issue 2, 2016

[6.] Ipere Polu O.H Gntonu P.E et al (2008), A review of the disease burden, impact and prevention of typhoid fever in Nigeria West African J. Med. Jul; 27 (3); 127 - 33.

[7.] Joe Brown, Sandy C., et al (2011). Sanitation Hygiene and Enteric Infection in children Arch Dis child 1136 - 30528

[8.] John A Crump, Eric D Muitz, (2010), Global trends in typhoid and paratyphoid fever. Clinical infect Dis, 50 (2): $241-243$.

[9.] Microbewiki, (2014). Typhoid fever in China. Accessed on $28^{\text {th }}$ July, 2014.

[10.] Mohammed H, Mirjam B. (2001). Risk factors for Clinical typhoid fever in villages, South Sulanesi, Indonesia. Int. J. of trop Med. Vol. 4 Pg. 91-99.

[11.] Sameen Siddiqiu (1991), Epidemiologic Patterns and Control Strategic in Typhoid Fever. JPMA pg. (143 to 146).

[12.] Samuel Kariu K, (2008), Typhoid fever in sub-Saharan Africa: Challenge of diagnosis and management of infection J Infect Developing Countries 2 (6) 443 - 447.

[13.] Typhoid Vaccine: Who position paper No. 6 (2008) Weekly Epidemiological Report 83: 49 - 60. [14.] Wikipedia, (2014). Typhoid Fever. Retrieved 30 ${ }^{\text {th }}$ July, 2014. 